\begin{tabular}{|l|}
\hline $010-011$ \\
Noticias \\
y comentarios \\
PH42 - Febrero 2003 \\
\hline
\end{tabular}

quietudes, problemas y denuncias relacionadas tanto con la profesión como con el Patrimonio.

Asimismo se anunció la reunión que durante los dias 21 y 22 de febrero de 2003 celebrará el grupo de Arte Contemporáneo en el Museo Nacional-Centro de Arte Reina Sofía de Madrid.

Para atender la demanda de los profesionales el GEIIC programará congresos temáticos y especializados con objeto de poder profundizar y ampliar los conocimientos en determinados campos de la conservación. El primero de ellos está previsto celebrarlo en Vitoria sobre El estudio de los brocados aplicados y otras técnicas de ejecución.

En colaboración con la Universidad Complutense de Madrid y con el Instituto del Patrimonio
Histórico Español, el GEIIC está preparando un curso bajo el título El textil y la indumentaria: materias, técnicas y evolución, con el fin de sensibilizar al público sobre la diversidad de esta industria y el papel que ha representado en la historia como simbolo de rango social y desarrollo tecnológico. Para ello, se reunirá a varios especialistas que abordarán el tema desde un punto de vista histórico, teórico y práctico.

\section{Para cualquier información dirigirse a:}

Grupo Español del IIC (Secretaria)

Instituto del Patrimonio Histórico Español (IPHE)

Tel.: 915504517

ana.laborde@iphe.mcu.es

concha.cirujano@iphe.mcu.es

\title{
Asociaciones de conservadores-restauradores proponen crear una Federación Profesional
}

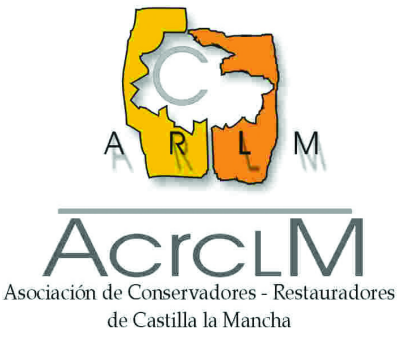

La Asociación de Conservadores-Restauradores de Castilla-La Mancha (www.iespana.es/ restauradorclm) participó en los Congresos del Grupo Español del IIC y en el XIV Congreso de Restauración, anteriormente reseñados.

Las asociaciones profesionales presentes en el Congreso del GE-IIC mantuvieron tres reuniones de trabajo, en las que se subrayó la necesidad de unión de profesionales y la creación, por tanto, de una Federación de Asociaciones que nos represente a nivel nacional y europeo, para intentar unificar, aclarar y avanzar, sacando a la Restauración de esta sensación de caos, en la que parece que estamos cada vez más perdidos.

Se constató el penoso nivel laboral en Restauración en Castilla-La Mancha y la inexistencia de profesionales en la Administración Autonómica, como muestra de una irresponsabilidad política generalizada en España, en temas relacionados con la Restauración y los criterios de intervención en Patrimonio.

En el XIV Congreso de Restauración se trasladó el esfuerzo de nuestra asociación por unirnos los titulados por las Escuelas Superiores de Conservación y Restauración de Bienes Culturales y de las licenciaturas en Bellas Artes, abandonadas en la Reforma Universitaria de 1990, tras la cual su especialidad de Restauración es definida por el Consejo de Universidades como una "intensificación académica".

Manifestamos a los asistentes al Congreso la intención de olvidar nuestras diferencias y que los de la Escuela y los de Bellas Artes estamos unidos. No nos sirven para nada las viejas rencillas, fomentadas en ambos lados, en las que el primer día de clase conociamos a Cesare Bran- di y a partir de ahí, día a día, se nos daba una tacita de odio al contrario.

La realidad es que tenemos dos vías claras para acceder a una licenciatura:

Una, es la que nos deja el R.D. 1387/1991 para la creación de un segundo ciclo, de licenciatura no universitaria, que seguiría permitiendo una enseñanza minoritaria, en la que se podría seguir manteniendo la enseñanza con quince alumnos por especialidad. Creemos que éste ha sido un punto fundamental para la enseñanza de la Restauración y es algo a tener en cuenta. En la actualidad es una Diplomatura a la que le faltaria un cuatrimestre para Licenciatura, es decir, que podria convertirse fácilmente en una Licenciatura no universitaria, de cuatro años.

La otra opción es una nueva licenciatura universitaria. La ventaja tampoco es despreciable, porque podríamos tener acceso al tercer ciclo, el tan deseado Doctorado, y desde luego las posibilidades que ofrece la Universidad en cuanto a investigación son dignas de tener en cuenta.

Estas son las dos posibilidades reales, una es más fácil que la otra. Éste es el punto de debate en el que nos encontramos. La vía del R.D. 1387/1991, que parece más sencilla y esperar tiempos mejores para que la Universidad nos abra las puertas, o seguir insistiendo, intentando demostrarle a la Universidad que nuestra enseñanza puede llegar a ser rentable económicamente. Porque, no nos engañemos mas, éste es el tema.

El Consejo de Universidades ha venido a complicar más las cosas creando la vía de los títulos propios. Estos títulos propios NO tienen validez mas que en el campus que se imparte. 
Si realmente los que pueden, los que dirigen estos títulos propios en restauración, quisieran solucionar esto, los veríamos batallando por los Congresos de Restauración unidos, diciendo lo mismo y quejándose de la poca asistencia que da la Universidad para su proyecto. Pero no, los vemos por separado; donde va uno no va el otro; y cada cual quiere vender su proyecto curricular no sabemos bien a quien.

Realmente el Consejo de Universidades ha abierto una gravísima brecha en nuestra profesión, porque los títulos propios se han creado para crear la sana competencia entre las distintas Universidades, para intentar captar alumnos con ofertas fantásticas y únicas de formación, pero nos ha pillado en medio, a los Restauradores y al Patrimonio.

Hubo polémica en Valencia de la que salió un "manifiesto" que debemos apoyar todos, porque no es más que el grito desesperado de una profesión que está harta de pedir reconocimiento a la propia administración que la titula; Y hubo polémica en Valladolid porque después de plantear todo esto y pedir que no hubiera interferencias mal intencionadas, para que pudiéramos intentarlo de nuevo desde una nueva perspectiva, abierta y transparente, se publicó un artículo en la Prensa de Valladolid hablando de la existencia de un único departamento especializado en restauración en una Universidad española.

Existe una posible solución, si existe alguna Universidad -neutral- que se preste a coordinar unas reuniones de trabajo para crear una licenciatura en Restauración. Presentar un proyecto curricular unificado al Consejo de Universidades, contando con todas las Instituciones en las que hay presencia de restauradores, con las Escuelas y con las asociaciones de profesionales. Es la única manera.

Pasearse por los Congresos unidos, y diciendo lo mismo, puede ser el primer paso para que nos creamos realmente que los profesionales dedicados a la enseñanza de la Restauración, desde la Universidad y las Escuelas Superiores, están luchando por nuestra profesión.

El debate está servido y si la propia Universidad no es capaz de arbitrar en este problema siendo plural, autocrítica y realista creo que es motivo suficiente para que no queramos - ni debamosestar dentro de ella y continuar como estamos, con los Doctorados en Historia del Arte y Bellas Artes, y con una única titulación reconocida, aunque sea a nivel de Diplomatura: la de las Escuelas Superiores de Conservación y Restauración. Quiero agradecer a la organización de los dos Congresos la oportunidad de debate que han ofrecido, pero es de esperar que en un futuro se coordinen un poco mejor, en tiempos, porque dos Congresos en una semana no hay cuerpo que lo aguante.

\section{Elena García Gayo}

Presidenta de la ACRCLM
Comunicado de la Comisión para la Creación de la Federación de Asociaciones de ConservadoresRestauradores de España

Algunas de las asociaciones de conservadores y restauradores de España, impulsadas por el GEIIC, nos hemos reunido con ocasión del $1^{\text {er }}$ Congreso para considerar la posibilidad de formar una Federación.

Las asociaciones que hemos estado presentes en esta primera reunión son las siguientes:

ACRA > Asociación de Conservadores-Restauradores de Aragón.

ACRLM > Asociación de Conservadores-Restauradores de Castilla-La Mancha.

ACRACV > Asociación de Conservadores -Restauradores de Arte de la Comunidad Valenciana.

GTCRC > Grup Tècnic, associació professional de Conservadors-Restauradors de béns culturals de Catalunya, en nombre propio y en representación de ARCC, Associació de Restauradors-Conservadors de Catalunya.

AKOBE > Asociación Cultural para la Conservación-Restauración del Patrimonio Histórico Artístico.

COLBBAA > Colegio Oficial de Doctores y Licenciados en Bellas Artes de Andalucía.

La voluntad de todas ellas es que se incorporen a la Comisión el mayor número de asociaciones de profesionales de la Conservación y la Restauración en España.

De las intervenciones que se han llevado a cabo durante el Congreso se desprende también la necesidad de que los profesionales que trabajamos en la Conservación y Restauración del patrimonio nos agrupemos para coordinar nuestros esfuerzos y dirigirlos conjuntamente para la obtención de los objetivos siguientes:

\section{Defender el ejercicio profesional.}

2. Proponer una formación única de nivel universitario superior, con equiparación curricular y homologación europea.

3. Establecer un código deontológico.

4. Impulsar el desarrollo de los reglamentos de las Leyes del Patrimonio en España, tanto los de la Ley estatal como los de las leyes de las distintas comunidades autónomas.

5. Fomentar el conocimiento de la profesión y la presencia mediática de la misma para evidenciar la necesidad de la regulación del ejercicio profesional.

6. Impulsar un cuerpo facultativo superior de técnicos en restauración para instituciones públicas.

Este primer listado de objetivos es un punto de partida para la elaboración de un documento de trabajo que deberá ser la base de la futura creación de la federación de asociaciones de profesionales de la Conservación-Restauración.

Desde este foro alentamos a todos los ConservadoresRestauradores que no pertenezcan a ninguna asociación a asociarse a alguna de ellas.

Valencia, 27 de noviembre de 2002 